\title{
A CHARACTERIZATION OF THE JACOBSON RADICAL IN TERNARY ALGEBRAS
}

\author{
HYO CHUL MYUNG
}

\begin{abstract}
The Jacobson radical $\operatorname{Rad} T$ for a ternary algebra $T$ is characterized as one of the following: (i) the set of properly quasi-invertible elements in $T$; (ii) the set of $x \in T$ such that the principal right ideal $\langle x T T\rangle$ or left ideal $\langle T T x\rangle$ is quasi-regular in $T$; (iii) the unique maximal quasi-regular ideal in $T$; (iv) the set of $x \in T$ such that $\operatorname{Rad} T^{(x)}=T^{(x)}$. We also obtain ternary algebraanalogs of characterization of the radicals of certain subalgebras in an associative algebra.
\end{abstract}

1. Introduction. Let $\Phi$ be a commutative ring with identity. A ternary algebra ( $\tau$-algebra) over $\Phi$ is defined as a unital $\Phi$-module $T$ with a trilinear composition $(x, y, z) \rightarrow\langle x y z\rangle$ satisfying

$$
\langle\langle x y z\rangle u v\rangle=\langle x\langle y z u\rangle v\rangle=\langle x y\langle z u v\rangle\rangle .
$$

In case $\Phi=Z, \tau$-algebras are $\tau$-rings defined by Lister [1], who extensively studies ring-theoretic structures for $\tau$-rings, in particular, a module theory leading up to the Jacobson radical. Lister defines the Jacobson radical, $R(T)$, for a $\tau$-ring $T$ to be the intersection of kernels of its irreducible modules and shows that $R(T)$ is the intersection of all maximal modular ideals in $T$ [1, Theorem 9]. Let $T$ now be a $\tau$-algebra. For $a \in T$, we denote by $T^{(a)}$ the algebra formed by setting

$$
x \cdot y=\langle x a y\rangle
$$

on the module $T$. Then, by (1), $T^{(a)}$ is an associative algebra. An element $x \in T$ is called properly quasi-invertible (p.q.i.) in $T$ if it is quasi-invertible (q.i.) in $T^{(a)}$ for all $a \in T$.

Definition. For a $\tau$-algebra $T$, the Jacobson $\operatorname{radical}, \operatorname{Rad} T$, of $T$ is the set of all p.q.i. elements in $T$.

In this paper we establish $\tau$-algebra-analogs of characterization of the radical in associative or Jordan algebras. Specifically, we will show that $\operatorname{Rad} T$ is an ideal of $T$ and coincides with the following: (i) the set of

Presented to the Society, April 6, 1972 under the title $A$ characterization of the Jacobson radical in ternary rings; received by the editors April 28, 1972 and, in revised form, July 24, 1972.

AMS (MOS) subject classifications (1970). Primary 16A21.

Key words and phrases. $\tau$-algebra, Jacobson radical, enveloping algebra, properly quasi-invertible, quasi-regular, Jordan triple system.

(c) American Mathematical Society 1973 
$x \in T$ such that $\langle x T T\rangle$ (or $\langle T T x\rangle$ ) is a quasi-regular right (or left) ideal in $T$; (ii) $\bigcap_{x \in T} \operatorname{Rad} T^{(x)}$; (iii) the set of $x \in T$ such that $\operatorname{Rad} T^{(x)}=T^{(x)}$. As applications of this we obtain $\tau$-algebra-analogs of such results as Theorems 4, 6, and 7 given by McCrimmon in [3]. Also, the present radical $\operatorname{Rad} T$ coincides with $R(T)$ if $T$ is a $\tau$-ring of Lister.

$\tau$-algebras have been called associative triple systems of 1 st kind by Loos [2], who has obtained analogous results for associative triple systems of 2 nd kind, defined by the rule

$$
\langle\langle x y z\rangle u v\rangle=\langle x\langle u z y\rangle v\rangle=\langle x y\langle z u v\rangle\rangle .
$$

The basic example of the 1st kind (or $\tau$-algebra) is a subspace of an associative algebra that is closed relative to $\langle x y z\rangle \equiv x y z$, while the basic example of the 2nd kind is a subspace of an associative algebra with involution, which is closed relative to $\langle x y z\rangle \equiv x \bar{y} z$. Setting $P(x) y=\langle x y x\rangle$, the 1 st and 2 nd kinds together with the quadratic mapping $P$ become Jordan triple systems (JTS) introduced by K. Meyberg [4]. Some of our present results have been proved for JTS in [4] where its proofs use complicated identities.

2. Preliminaries. Throughout we assume $T$ denotes a $\tau$-algebra over $\Phi$. Any $\tau$-algebra $T$ can be imbedded into an associative algebra $A=$ $T+T^{2}$ regarded as a $\tau$-algebra [1, p. 39]. Then $A$ is called an enveloping algebra of $T$ and if $A=T \oplus T^{2}$, the imbedding is called direct. A $\tau$-algebra always possesses a direct enveloping algebra $\left[1\right.$, p. 38]. If $A=T+T^{2}$ and $a \in A$, we also denote by $A^{(a)}$ the a-homotope of $A$. Thus if $a \in T, T^{(a)}$ is a subalgebra of $A^{(a)}$. Recall $x \in A$ is q.i. in $A$ if $x+y=x y=y x$ for some $y \in A$.

For $x, y \in T$ we introduce the useful operator

$$
B(x, y) \equiv \mathrm{Id}-L(x, y): z \mapsto\langle x y z\rangle
$$

for $z \in T$. Then we directly see from (1) the following:

$$
\begin{aligned}
B(\langle x y z\rangle, u) & =B(x,\langle y z u\rangle), \\
B(x, y) B(x,-y) & =B(x,\langle y x y\rangle) .
\end{aligned}
$$

If $x, y \in T$ and $y$ is a quasi-inverse of $x$ in $T^{(u)}$, then $x+y=\langle x u y\rangle$ and for all $z \in T$,

$$
\begin{aligned}
B(x, u) B(-y, z) v & =v+\langle y z v\rangle-\langle x u v\rangle-\langle x u\langle y z v\rangle\rangle \\
& =v+\langle y z v\rangle-\langle x u v\rangle-\langle(x+y) z v\rangle \\
& =B(x, u+z) v .
\end{aligned}
$$

Hence we have the Addition Formula: if $x, y \in T$ are quasi-inverses in $T^{(u)}, B(x, u) B(-y, z)=B(x, u+z)$ for all $z \in T$. 
LeMma 1. If $A=T \oplus T^{2}$, then, for $a, x \in T$, the following are equivalent :

(i) $x$ is q.i. in $T^{(a)}$;

(i') $a$ is q.i. in $T^{(x)}$;

(ii) $x$ is q.i. in $A^{(a)}$;

(ii') $a$ is q.i. in $A^{(x)}$;

(iii) $x a$ is q.i. in $A$;

(iii') ax is q.i. in $A$;

(iv) $B(x, a)$ is bijective on $T$;

(iv') $B(a, x)$ is bijective on $T$.

Proof. That (i) $\Rightarrow$ (ii) is obvious, and that (ii) $\Rightarrow$ (iii) is a result of McCrimmon [3, Proposition 1]. (iii) $\Rightarrow$ (iv): since $x a$ is q.i. in $A$, the left multiplication $L(1-x a)$ in $A$ is invertible on $A$ (if $A$ does not contain 1, adjoin an identity to $A$ ). Then $T$ and $T^{2}$ are invariant under $L(1-x a)$, and $B(x, a)$ is the restriction of $L(1-x a)$ to $T$. Noting that an element in $T^{2}$ is q.i. in $T^{2}$ if and only if it is q.i. in $A$ since the imbedding is direct, we have that $B(x, a)$ is still invertible on $T$. (iv) $\Rightarrow(\mathrm{i})$ : by surjectivity $B(x, a) y=-x$ for some $y \in T$, i.e., $x+y=\langle x a y\rangle$. But

$$
\begin{aligned}
B(x, a)\langle y a x\rangle & =\langle y a x\rangle-\langle x a\langle y a x\rangle\rangle=\langle y a x\rangle-\langle\langle x a y\rangle a x\rangle \\
& =\langle y a x\rangle-\langle x a x\rangle-\langle y a x\rangle=-\langle x a x\rangle \\
& =x+y-\langle x a(x+y)\rangle=B(x, a)(x+y)
\end{aligned}
$$

and so $x+y=\langle y a x\rangle$ too since $B(x, a)$ is injective. By symmetry we prove that $\left(\mathrm{i}^{\prime}\right) \Leftrightarrow\left(\mathrm{ii}^{\prime}\right) \Leftrightarrow\left(\mathrm{iii}^{\prime}\right) \Leftrightarrow\left(\mathrm{iv}^{\prime}\right)$. But that (iii) $\Leftrightarrow\left(\mathrm{iii}^{\prime}\right)$ is well known for associative algebras, and the proof is complete.

By symmetry in Lemma 1 in $x$ and $a$, we have a Symmetry Principle for $T$ :

$x$ is q.i. in $T^{(y)}$ if and only if $y$ is q.i. in $T^{(x)}$.

From this we get a Shifting Principle for $T$ :

$x$ is q.i. in $T^{\langle y z u\rangle)}$ if and only if $u$ is q.i. in $T^{\langle\langle x y z\rangle)}$,

since, by Lemma $1, x$ is q.i. in $T^{(\langle y z u\rangle)}$ if and only if $B(x,\langle y z u\rangle)=$ $B(\langle x y z\rangle, u)$ (by (2)) is bijective if and only if $u$ is q.i. in $T^{(\langle x y z\rangle)}$. Hence we obtain the Shifting Theorem for $T$ :

THEOREM 1. The following conditions for $x, y, z, u$ in a $\tau$-algebra $T$ are equivalent:

(i) $x$ is q.i. in $T^{(\langle y z u\rangle)}$;

(ii) $u$ is q.i. in $T^{\langle\langle x y z\rangle)}$;

(iii) $y$ is q.i. in $T^{\langle\langle z u x\rangle)}$;

(iv) $z$ is q.i. in $T^{(\langle u x y\rangle)}$. 
In view of the equivalence of (i), (ii), and (iii') in Lemma 1 we can state

Lemma 2. If $A=T \oplus T^{2}$, then $x \in T$ is p.q.i. in $T$ if and only if $x a$ and ax are q.i. in $T^{2}$ (or in $A$ ) for all $a \in T$.

3. Characterization of the radical. A subspace $V$ of $T$ is called a right ideal of $T$ in case $\langle V T T\rangle \subseteq V$, a left ideal in case $\langle T T V\rangle \subseteq V$, a medial ideal in case $\langle T V T\rangle \subseteq V$, and $V$ is called an ideal in $T$ if it is right, left, and medial. Thus a right (left) ideal $V$ in $T$ is a right (left) ideal in all $T^{(x)}$. A right ideal $V$ of $T$ is called (right) quasi-regular (q.r.) [1, p. 45] if $B(v, x) T=T$ for all $v \in V$ and all $x \in T$, and a q.r. left ideal is similarly defined. Noting that $V$ is (right) q.r. in all $T^{(x)}$, this condition is equivalent to that every $v \in V$ is p.q.i. in $T$.

THEOREM 2. For a $\tau$-algebra $T$, the radical $\operatorname{Rad} T$ is an ideal of $T$.

Proof. Let $x \in T$ be p.q.i. in $T$. Then $\alpha x$ is p.q.i. for $\alpha \in \Phi$ since all $B(\alpha x, y)=B(x, \alpha y)$ are bijective. Now, $\langle x y z\rangle$ are p.q.i. for all $y, z \in T$ since $B(\langle x y z\rangle, u)=B(x,\langle y z u\rangle)$, and all $\langle y x z\rangle$ are p.q.i. since all $B(y,\langle x z u\rangle)$ are bijective (Symmetry) and so are all $B(u,\langle y x z\rangle)$ (Shifting). From this, using Symmetry and Shifting Principles, we also see that all $\langle y z x\rangle$ are p.q.i. in $T$. Finally, let $z, u$ be p.q.i. in $T$ and let $x \in T$. By Symmetry $x$ is q.i. in $T^{(u)}$, and if $y$ is the quasi-inverse of $x$ in $T^{(u)}$ then by the Addition Formula $B(x, u) B(-y, z)=B(x, u+z)$. Since $B(x, u)$ and $B(-y, z)$ are bijective so is $B(x, u+z)$ for all $x \in T$. Thus the set of all p.q.i. elements in $T$ forms an ideal of $T$.

LEMMA 3. If $A=T \oplus T^{2}$, the following are equivalent :

(i) $x \in T$ is p.q.i. in $T$;

(ii) $x T$ is a q.r. right ideal in $T^{2}$;

(ii') $T x$ is a q.r. left ideal in $T^{2}$;

(iii) the principal right ideal $\langle x T T\rangle$ is q.r. in $T$;

(iii') the principal left ideal $\langle T T x\rangle$ is q.r. in $T$.

Proof. (i) $\Leftrightarrow$ (ii). This follows from Lemma 2. (i) $\Leftrightarrow$ (iii). If $x$ is p.q.i. in $T$, all $B(x,\langle y z u\rangle)=B(\langle x y z\rangle, u)$ are bijective and so by Theorem 2 all elements in $\langle x T T\rangle$ are p.q.i., i.e., $\langle x T T\rangle$ is q.r. in $T$. Conversely, if $\langle x T T\rangle$ is q.r. in $T$, all $B(\langle x y z\rangle, u)=B(x,\langle y z u\rangle)$ are bijective. Then by (3) all $B(x, t) B(x,-t)$ and $B(x,-t) B(x, t)$ are bijective, so all $B(x, t)$ are too. Since (i) is left-right symmetric, we get (i) $\Leftrightarrow\left(\mathrm{ii}^{\prime}\right) \Leftrightarrow\left(\mathrm{iii}^{\prime}\right)$, and the proof is complete.

In view of Theorem 2 and Lemma 3(i), (iii), (iii'), we have the following analogous result of associative algebras (also see [1]):

COROLlaRY 1. $\operatorname{Rad} T$ is a q.r. ideal in $T$ and contains all q.r. right ideals and q.r. left ideals in $T$. Hence $\operatorname{Rad} T$ is the unique maximal q.r. ideal in $T$. 
REMARK. In case $T$ is a $\tau$-ring, this corollary has been proved for the radical $R(T)$ at characteristic $\neq 2$ by Lister [1, Theorem 10]. Hence $\operatorname{Rad} T$ coincides with the radical $R(T)$ defined by Lister.

Corollary 2. If $A=T \oplus T^{2}$, then

$$
\operatorname{Rad} T=\left\{x \in T \mid x T \subseteq \operatorname{Rad} T^{2}\right\} .
$$

Proof. This follows from Lemma 3 and the well-known fact for associative algebras.

THEOREM 3. For a $\tau$-algebra $T$, we have

$$
\begin{aligned}
\operatorname{Rad} T & =\{x \in T \mid\langle x T T\rangle \text { or }\langle T T x\rangle \text { is q.r. in } T\} \\
& =\left\{x \in T \mid \operatorname{Rad} T^{(x)}=T^{(x)}\right\} \\
& =\bigcap_{a \in T} \operatorname{Rad} T^{(a)} .
\end{aligned}
$$

Proof. $\operatorname{Rad} T$ equals the first set by Lemma 3(i), (iii), (iii'). If $x \in$ $\operatorname{Rad} T, x$ is q.i. in all $T^{(\langle z x z\rangle)}$ and by Symmetry all $\langle z x z\rangle$ are q.i. in $T^{(x)}$; so are all $z$ in $T^{(x)}$ since $\langle z x z\rangle=z^{2}$ in $T^{(x)}$. Hence $\operatorname{Rad} T^{(x)}=T^{(x)}$. Conversely, if $\operatorname{Rad} T^{(x)}=T^{(x)}$, all $y$ are q.i. in $T^{(x)}$ and by Symmetry $x$ is p.q.i. in $T$, i.e., $x \in \operatorname{Rad} T$. Thus $\operatorname{Rad} T$ equals the second set. If $x \in \bigcap \operatorname{Rad} T^{(a)}, x$ is q.i. in all $T^{(a)}$ and is p.q.i. in $T$, while if $x$ is p.q.i. in $T$, so is $x$ in all $T^{(a)}$ since the $y$-homotope of $T^{(a)}$ is $T^{(\langle a y a\rangle)}$. Hence $\operatorname{Rad} T=\bigcap \operatorname{Rad} T^{(a)}$ This completes the proof.

4. Applications. In this section we use the previous results to characterize the radicals of certain $\tau$-subalgebras of $T$. A left (or right, or leftright) ideal $V$ of $T$ is also a left (or right, or two-sided) ideal in all $T^{(x)}$, and $V^{(a)}$ for every $a \in T$ is a left, right, or two-sided ideal of $T^{(a)}$, according as $V$ is left, right, or left-right. Hence, in any case, $V$ is a strict inner ideal in all $T^{(x)}$ and $V^{(a)}$ is strictly inner in $T^{(a)}$. We now recall that if $K$ is a strict inner ideal of an alternative algebra $B$ and $x \in K$ is q.i. in $B$, then $x$ is q.i. in $K$ [3, p. 571]. If $U$ is an ideal in a $\tau$-ring $T$, Lister [1, p. 46] proves that $R(U)=U \cap R(T)$. We extend this to left-medial (or medial-right) ideals in a $\tau$-algebra $T$ and give another proof of this as well.

THEOREM 4. If $V$ is a left-medial (or medial-right) ideal in $T$, then $\operatorname{Rad} V=V \cap \operatorname{Rad} T$.

Proof. We proceed as in [3, Theorem 3]. If $x \in V \cap \operatorname{Rad} T, x$ is q.i. in all $T^{(a)}$ and so q.i. in all $V^{(a)}$ since $V^{(a)}$ is a left ideal in $T^{(a)}$. Hence $x$ is p.q.i. in $V$ and so $x \in \operatorname{Rad} V$. Conversely, if $x \in \operatorname{Rad} V, x$ is q.i. in $V^{(\langle a x a\rangle)}$ (so in $T^{(\langle a x a\rangle)}$ ) for all $a \in T$ since $V$ is medial. Hence, by Symmetry, each $\langle a x a\rangle$, which is the square of $a$ in $T^{(x)}$, is q.i. in $T^{(x)}$. Thus all $a$ are q.i. in $T^{(x)}$ and by Symmetry $x \in \operatorname{Rad} T$. 
For left or right ideals of $T$, we obtain a result similar to [3, Theorem 4]:

THEOREM 5. If $V$ is a left ideal in $T$, then

$$
\begin{aligned}
\operatorname{Rad} V & =\{z \in V \mid\langle V V z\rangle \subseteq \operatorname{Rad} T\} \\
& =\{z \in V \mid\langle T V z\rangle \subseteq \operatorname{Rad} T\} .
\end{aligned}
$$

Proof. We only prove the second equality and the other case is entirely similar. Let $z \in V$ be such that $\langle T V z\rangle \subseteq \operatorname{Rad} T$. Then every $x \in$ $\langle T V z\rangle(\subseteq V)$ is q.i. in all $T^{(a)}$ and since $V^{(a)}$ is a left ideal of $T^{(a)}, x$ is q.i. in all $V^{(a)}$, so in particular $\langle V V z\rangle$ is a q.r. left ideal of $V$. Hence by Lemma $3, z \in \operatorname{Rad} V$. Conversely, if $z \in \operatorname{Rad} V$, let $a$ be any element of $V$. Then $z$ is q.i. in $V^{\langle\langle x y a\rangle)}$ (so in $T^{(\langle x y a\rangle)}$ ) for all $x, y \in T$, and so, shifting, all $x$ are q.i. in $T^{\langle(y a z\rangle)}$. Hence by Symmetry $\langle y a z\rangle$ is q.i. in all $T^{(x)}$ and $\langle y a z\rangle \in$ $\operatorname{Rad} T$. Thus $\langle T V z\rangle \subseteq \operatorname{Rad} T$.

As usual, a subspace $V$ of $T$ is called an inner ideal of $T$ if $\langle a T a\rangle \subseteq V$ for all $a \in V$. Thus all left, right ideals and the subspaces $\langle x T x\rangle$ are inner ideals in $T$. Furthermore, if $V$ is an inner ideal in $T$ then $V^{(a)}$ is a strict inner ideal in $T^{(a)}$ for all $a \in T$ since $\langle v a v\rangle=v^{2}$ in $V^{(a)}$ for $v \in V$. The following characterization of the radical is applied for all these examples.

THEOREM 6. If $V$ is an inner ideal in $T$, then

$$
\operatorname{Rad} V=\{z \in V \mid\langle a z a\rangle \in \operatorname{Rad} T \text { for all } a \in V\} .
$$

Proof. If $z \in V$ and $\langle a z a\rangle \in \operatorname{Rad} T$ for all $a \in V,\langle a z a\rangle \in V$ is q.i. in all $T^{(x)}$ and so is q.i. in all $V^{(x)}$ since $V^{(x)}$ is strictly inner in $T^{(x)}$. In particular, all $\langle a z a\rangle$ are q.i. in $V^{(z)}$, but $\langle a z a\rangle=a^{2}$ in $V^{(z)}$ and so all $a$ are q.i. in $V^{(z)}$ (again recall $V^{(z)}$ is strictly inner in $T^{(z)}$ ). Thus by Symmetry $z \in \operatorname{Rad} V$. The converse is the same as in [3, Theorem 7].

An element $e \in T$ is called an idempotent if $\langle e e e\rangle=e^{3}=e$. If $z \in \operatorname{Rad}\langle e T e\rangle$ then $z=\langle e x e\rangle$ for some $x \in T$ and so $z=\langle e\langle e z e\rangle e\rangle \in \operatorname{Rad} T$ by Theorem 6 . Hence we obtain

COROllary. If e is an idempotent in $T$, then

$$
\operatorname{Rad}\langle e T e\rangle=\langle e T e\rangle \cap \operatorname{Rad} T .
$$

5. Relations with Jordan triple systems. For a $\tau$-algebra $T$, let $T_{J}$ denote the JTS formed from $T$ by setting $P(a) x=\langle a x a\rangle$. From $T^{(a)}$, we also form a quadratic Jordan algebra $T^{(a)+}$ by setting $P_{a}(x)=P(x) P(a)$ and $x^{2(a)}=P(a) x$, and it is well known that $\operatorname{Rad} T^{(a)}=\operatorname{Rad} T^{(a)+}$. Thus, in view of Theorem 3 and [4], we have

THEOREM 7. For a $\tau$-algebra $T, \operatorname{Rad} T=\operatorname{Rad} T_{J}$. 
As usual, $a \in T$ is called von Neumann regular if $a=\langle a x a\rangle$ for some $x \in T$, and $b \in T$ is called trivial if $\langle b T b\rangle=0$. While one-sided ideals or ideals in $T$ are different from those in $T_{J}, T$ and $T_{J}$ have the same inner ideals, idempotents, trivial elements, and von Neumann regularity. Hence, from Theorem 7 and the known result [4] for a JTS, we can state

THEOREM 8. If $T$ is a $\tau$-algebra satisfying the d.c.c. on inner ideals, then the following are equivalent:

(i) $T$ is semisimple;

(ii) $T$ is von Neumann regular;

(iii) $T$ contains no nonzero trivial elements.

A proof of this for a JTS uses complicated identities [4], but some parts of the proof for $T$ are considerably shorter. For example, to show that if $a \in T$ is trivial then $a \in \operatorname{Rad} T$, we simply observe that every element of $\langle a T T\rangle$ is trivial and so $\langle a T T\rangle$ is q.r. in $T$. Next, to see that if $T$ is von Neumann regular then it is semisimple, let $z \in \operatorname{Rad} T$. Then $z=\langle z a z\rangle$ for some $a$, so $B(z, a) z=0$; but since $B(z, a)$ is invertible, $z=0$.

Finally, let $B$ be an associative algebra. Then $B$ becomes a $\tau$-algebra on iteration of its product, which we denote by $B_{r}$. Let $B^{+}$and $B_{J}$, respectively, denote the quadratic Jordan algebra and the JTS formed from $B$ in the usual manner. Then from Theorems 3 and 7 we obtain

THEOREM 9. If $B$ is an associative algebra, then

$$
\operatorname{Rad} B=\operatorname{Rad} B^{+}=\operatorname{Rad} B_{\tau}=\operatorname{Rad} B_{J} \text {. }
$$

ACKNOWLEDGEMENT. The author wishes to express his gratitude to the referee for his kind advice and many useful suggestions, especially for suggesting the operator $B(x, y)$ by which the author could remove the condition of characteristic $\neq 2$ in the original proofs.

\section{REFERENCES}

1. W. G. Lister, Ternary rings, Trans. Amer. Math. Soc. 154 (1971), 37-55. MR 42 \#7716.

2. O. Loos, Assoziative Tripelsysteme, Manuscripta Math. 7 (1972), 103-112.

3. K. McCrimmon, A characterization of the Jacobson-Smiley radical, J. Algebra 18 (1971), 565-573. MR 43 \#3318.

4. K. Meyberg, Lectures on algebras and triple systems, Lecture Notes, University of Virginia, Charlottesville, Va., 1972.

Department of Mathematics, University of Northern Iowa, Cedar Falls, IOWA 50613 